\title{
CONSUMO DE MATERIA SECA PROVENIENTE DE DIFERENTES ESPECIES FORRAJERAS EN CABRAS EN COSTA RICA ${ }^{1}$
}

\author{
Jorge Alberto Elizondo-Salazar ${ }^{2 \varpi}$
}

\section{RESUMEN}

Las explotaciones caprinas han jugado un papel muy importante en muchas culturas del mundo y en los últimos años, su población se ha visto aumentada debido a una creciente e importante demanda de alimentos ricos en proteína, consecuencia del crecimiento de la población humana y de una mayor exigencia en la calidad de los productos animales que consume. Dicha demanda ha sido en parte satisfecha con caprinos que, gracias a su condición de rumiantes, son capaces de procesar forrajes y residuos de cosechas no aptos para consumo humano, convirtiéndolos en alimentos de alta calidad nutricional. Sin embargo, con la apertura de los mercados y la globalización mundial, cualquier actividad productiva debe ser manejada eficientemente y la explotación caprina no escapa a esta exigencia. Para aumentar su producción hay que comprender que las cabras requieren de una alimentación rica en nutrientes presentes en la materia seca de los alimentos, pero cuando se considera este indicador en cabras, el rango de valores es poco conocido, por lo que el presente trabajo tiene el objetivo de presentar, a través de una revisión de literatura, los consumos de materia seca en cabras, provenientes de diferentes especies forrajeras, que reportan las diferentes investigaciones científicas llevadas a cabo en nuestro país. Los consumos de materia seca reportados en la literatura oscilan entre 250 y 1200 g por animal por día. Considerando la baja calidad nutricional de muchos de nuestros forrajes, es muy probable que con estos consumos los animales no estén llenando sus necesidades nutricionales, por lo que se deben buscar alternativas alimenticias y medidas de manejo que logren aumentar los consumos de materia seca y por ende, el consumo de nutrimentos en estos animales, de manera que puedan mejorar tanto su desempeño productivo como reproductivo.

Palabras clave: Nutrición, Capra hircus, forrajes, pequeños rumiantes

'Proyecto ED-2746 inscrito en la Vicerrectoría de Acción Social de la Universidad de Costa Rica.

${ }^{2}$ Universidad de Costa Rica, Facultad de Ciencias Agroalimentarias. Estación Experimental Alfredo Volio Mata.

$\triangle$ Autor para correspondencia: jorge.elizondosalazar@ucr.ac.cr

Recibido: 1 octubre 2018

Aceptado: 26 noviembre 2018 


\section{ABSTRACT}

Dry matter intake from different forage species in goats in Costa Rica. Goats have played a very important role in many cultures of the world and in recent years, their population has increased due to a growing and important demand for protein rich foods, because of human population growth and of a greater demand on the quality of animal products they consume. This demand has been partly satisfied with goats that, thanks to their condition as ruminants, are able to process fodder and crop residues not suitable for human consumption, converting them into foods of high nutritional quality. However, with the opening of markets and globalization, any productive activity must be managed efficiently, and goat production does not escape this demand. To increase their production, it is necessary to understand that goats require a diet rich in nutrients present in the dry matter fraction of feeds, but when this indicator is considered in goats, the range of values is not well known, thus the present work has the objective of presenting, through a literature review, dry matter consumption in goats, from different forage species, that has been reported in different scientific investigations carried out in Costa Rica. Dry matter intake reported in the literature varies between 250 and $1200 \mathrm{~g}$ per animal per day. Considering the low nutritional quality of many of our forages, it is very likely that with these kind of intake animals are not filling their nutritional needs, which is why producers should look for supplement alternatives and management measures that increase dry matter intake and therefore the consumption of nutrients in these animals, so that they can improve both their productive and reproductive performance.

Key words: Nutrition, Capra hircus, forages, small ruminant 


\section{INTRODUCCIÓN}

Las cabras se explotan en todo el mundo por su leche, su carne y su piel, con un grado de especialización muy variado, desde explotaciones extensivas hasta estructuras muy intensivas en países industrializados. En Costa Rica, el Censo Agropecuario realizado en el año 2014, indica que para ese momento había en el país 2348 fincas dedicadas a la actividad caprina, con un total de 12852 animales ubicados en fincas, más 1251 cabras que se ubican en espacios que no son fincas, conformando el hato caprino nacional con 14103 animales, donde las hembras representan el $80,4 \%$ y los machos el 19,6\%. La provincia con la cantidad más elevada de caprinos es Alajuela con 3352, seguida por San José con 2228, sumando en conjunto el $43,4 \%$ del total de estos animales. Los cantones con la mayor cantidad de caprinos son San Carlos, Pérez Zeledón y Santa Cruz donde se encuentra prácticamente la cuarta parte de estos animales (24,2\%) (INEC, 2015).

Durante muchos años, las cabras han sido particularmente útiles para el hombre, esencialmente por su adaptabilidad a condiciones ambientales variables. En muchas partes de los trópicos, la baja disponibilidad de nutrimentos y las difíciles condiciones del medio son de tal magnitud que los caprinos resultan los animales más recomendables para dichas regiones (Devendra, 1981). Sin embargo, los niveles productivos en muchas ocasiones son bajos, debido a la pobre genética de los animales, a deficiencias en el manejo, a la influencia del clima, y principalmente a una deficiente alimentación. Por esta razón se debe tener un conocimiento más detallado del comportamiento alimenticio de estos animales, para mejorar la ingesta de nutrientes, que es el mayor factor que limita la producción, y poder así buscar alternativas alimenticias que logren satisfacer las necesidades nutricionales en todas las etapas.

El consumo de materia seca (MS) en las cabras es determinado por un proceso de saciedad, que obedece a una serie de señales fisiológicas que reaccionan a la composición del alimento y a su procesamiento en el organismo del animal. Algunas de estas señales son la sensación de llenado en el rumen, la concentración de los productos de la fermentación, la concentración de nutrientes en la dieta y el requerimiento nutricional (Baumont et al., 2000).

En adición a estos y otros factores fisiológicos involucrados en el consumo, las características de los forrajes tienen también una gran influencia. El primer factor por considerar es el llenado físico que provoca la concentración de fibra detergente neutro (FDN). Allen (1996) considera 
que la FDN es el factor más determinante para estimar el consumo voluntario de materia seca; sin embargo, otros factores asociados afectan el llenado físico del animal como el tamaño de partícula, la frecuencia y eficiencia del masticado, la fragilidad de la partícula, la fracción indigestible de la FDN y la tasa de fermentación de la FDN digestible. Es necesario considerar que además de la concentración de FDN, el contenido de lignina en el forraje es igual de efectivo para disminuir el consumo (Jung y Allen, 1995).

Otras características genéticas de los forrajes tropicales que generalmente no se consideran en los análisis bromatológicos, pueden también tener un efecto negativo sobre el consumo, como por ejemplo los taninos, alcaloides, aceites, terpenos, cianógenos, inhibidores de la proteasa, sesquiterpenos, saponinas, aminoácidos no proteicos, péptidos tóxicos y ácidos orgánicos, entre otros (Sands, 1983).

A este posible efecto negativo en el consumo ocasionado por los compuestos secundarios, debe sumarse el contenido de humedad presente en el forraje. Este factor influye sobre el consumo de materia seca y la digestibilidad de la misma (Pasha et al., 1994). La hora del día en que se cosecha el forraje puede influir también sobre el consumo de materia seca, ya que se han encontrado variaciones en el contenido de carbohidratos no estructurales durante el día y a pesar de que esta variación puede ser pequeña, al comparar los cortes de la mañana y de la tarde, es suficiente para estimular un mayor consumo en los cortes realizados en la tarde, debido probablemente a un aumento en el contenido de carbohidratos no estructurales y a una mayor digestibilidad de los componentes de la planta (Fisher et al., 1999; Fisher et al., 2002, Burns et al., 2005).

La integración de todos estos y otros factores es muy importante para explicar el consumo de materia seca en cabras. En este aspecto, es importante considerar la amplitud del rango que se reporta en la literatura. Por ejemplo, Morand-Fehr (1981) reporta que en un total de 5500 registros de consumo diario en 130 cabras Alpinas que consumían pasto o heno y alimento balanceado durante los últimos seis meses de gestación, el consumo voluntario de las cabras podía variar de 0,82 a 2,13 kg de MS por día. Sands (1983) indica que se revisó la literatura relacionada con el consumo de MS en cabras y de 35 ensayos realizados en diferentes lugares del mundo, se observó que las cabras que consumieron gramíneas tropicales y plantas arbustivas, sin alimento balanceado, mostraron consumos entre 1,01 y $2,46 \%$ de su peso corporal. Estos valores muestran que existe una gran variabilidad en el consumo de materia 
seca proveniente de forrajes, especialmente cuando se considera que son muchos los factores que afectan el consumo.

\section{Estudios llevados a cabo en Costa Rica}

En Costa Rica algunas de las investigaciones realizadas desde los años 80's han evaluado el desempeño productivo en cabras alimentadas con diferentes fuentes forrajeras, cuantificándolo como producción láctea, ganancia de peso o consumo de materia seca.

Se han utilizado una gran variedad de forrajes (Cuadro 1) como gramíneas (Samur, 1984; Castro, 1989; Elizondo-Salazar, 2004b; Chacón, 2008; Herrera-Campos et al., 2009; VargasRodríguez, 2009; Rodríguez-Zamora y Elizondo-Salazar, 2012; Elizondo-Salazar, 2015; Salazar-Acosta y Elizondo-Salazar, 2017), arbustivas (Elizondo, 2004a, 2005; Herrera-Campos et al., 2009; Rodríguez-Zamora y Elizondo-Salazar, 2012), leguminosas (Gutiérrez, 1985; Rodríguez, 1989) y ensilajes (Vallejo, 1995; Chávez, 2005). En estos trabajos se ha logrado evaluar la selectividad y el consumo tanto en cabras secas como lactantes. A pesar de todos estos estudios, no ha sido posible establecer un parámetro de consumo que pueda ser aplicado en el país, incluso se presenta una gran variabilidad entre especies forrajeras similares.

Los primeros trabajos realizados en cabras se concentraron en evaluar la preferencia de estos animales por las distintas especies forrajeras. Samur (1984) evaluó el consumo en 24 cabras lactantes mestizas, de 32 a 119 días de lactancia y con un peso promedio de $36,7 \mathrm{~kg}$. Utilizó una ración de pasto de king grass (Pennisetum purpureum) suplementada con banano verde (Musa sp.) o maduro y poró (Erythrina poeppigiana). El alimento se ofreció de manera que los animales consumieran banano y poró mezclados o separados con una diferencia de ofrecimiento de una a dos horas. En este caso, la forma de suministrar el banano y el poró no influyó sobre el consumo de king grass que en promedio fue de $473 \mathrm{~g} \mathrm{MS}$.animal $^{-1} . \mathrm{d}^{-1}$.

Luego Castro (1989) continuó con esta línea de trabajo, midiendo el consumo y la producción láctea de doce cabras nubiano-criollo con 65 días de lactancia y con 47,3 kg de peso promedio. En este caso, a la dieta a base de pasto se le añadió plátano (Musa sp.) y poró combinados en diferentes proporciones. Este autor determinó un consumo para el king grass de $900 \mathrm{~g}$ MS.animal ${ }^{-1} \cdot \mathrm{d}^{-1}$, y encontró que el mayor consumo de pasto estaba asociado con la menor proporción de poró y plátano. 
Cuadro 1. Consumo de materia seca (g/animal/d y porcentaje del peso vivo) en cabras consumiendo diferentes tipos de plantas forrajeras.

\begin{tabular}{|c|c|c|c|c|}
\hline Material forrajero & Forraje & g/animal/d & Peso vivo, \% & Autor \\
\hline Amapola & $E$ & 542,00 & 1,09 & Vallejo (1995) \\
\hline $\begin{array}{l}\text { Cratylia + sorgo } \\
\text { negro+melaza }\end{array}$ & $E$ & 515,00 & 1,26 & Chávez (2005) \\
\hline Estrella africana fresca & G & 760,00 & 1,52 & Herrera-Campos et al. (2009) \\
\hline Estrella africana fresca & G & 930,00 & 2,51 & $\begin{array}{l}\text { Zamora-Rodríguez y Elizondo-Salazar } \\
\text { (2012) }\end{array}$ \\
\hline $\begin{array}{l}\text { Estrella africana } \\
\text { deshidratada }\end{array}$ & G & 760,00 & 2,05 & $\begin{array}{l}\text { Zamora-Rodríguez y Elizondo-Salazar } \\
\text { (2012) }\end{array}$ \\
\hline Estrella africana fresca & G & 780,00 & 1,37 & Elizondo-Salazar (2015) \\
\hline Estrella africana+alimento & G & 930,00 & 1,63 & Elizondo-Salazar (2015) \\
\hline Estrella africana fresca & G & 491,03 & 0,94 & $\begin{array}{l}\text { Salazar-Acosta y Elizondo-Salazar } \\
(2017)\end{array}$ \\
\hline Jocote & $E$ & 394,00 & 0,79 & Vallejo (1995) \\
\hline King grass & $\mathrm{G}$ & 473,00 & 1,29 & Samur (1984) \\
\hline King grass & G & 900,00 & 1,90 & Castro, (1989) \\
\hline King grass & $\mathrm{G}$ & 250,92 & 0,57 & Chacón (2008) \\
\hline King grass & $G$ & 977,00 & 1,95 & $\begin{array}{l}\text { Vargas-Rodríguez y Carvajal-Villalta } \\
\text { (2018) }\end{array}$ \\
\hline Madero negro & $\mathrm{L}$ & 860,00 & 2,02 & Rodríguez (1989) \\
\hline Morera fresca & $A$ & 630,00 & 1,26 & Herrera-Campos et al. (2009) \\
\hline Morera fresca & $A$ & 594,00 & 1,20 & Vallejo (1995) \\
\hline Morera fresca & $A$ & 737,32 & 1,94 & Elizondo-Salazar (2004a) \\
\hline Morera fresca & A & 1170,00 & 3,16 & $\begin{array}{l}\text { Zamora-Rodríguez y Elizondo-Salazar, } \\
\text { (2012) }\end{array}$ \\
\hline Morera deshidratada & $A$ & 1120,00 & 3,02 & $\begin{array}{l}\text { Zamora-Rodríguez y Elizondo-Salazar } \\
\text { (2012) }\end{array}$ \\
\hline Pasto prodigioso & G & 342,03 & 0,86 & Vargas-Rodríguez (2009) \\
\hline Poró & L & 1200,00 & 2,82 & Rodríguez (1989) \\
\hline Sorgo negro forrajero & G & 690,00 & 1,38 & Herrera-Campos et al. (2009) \\
\hline Sorgo negro forrajero & G & 371,00 & 0,98 & Elizondo-Salazar (2004b) \\
\hline Sorgo negro forrajero & G & 342,03 & 0,90 & Elizondo-Salazar (2005) \\
\hline $\begin{array}{l}\text { Sorgo negro } \\
\text { forrajero+ramio }\end{array}$ & $M$ & 625,88 & 1,65 & Elizondo-Salazar (2005) \\
\hline Forraje de maíz fresco & G & 490,00 & 0,86 & Elizondo-Salazar (2015) \\
\hline $\begin{array}{l}\text { Forraje de maíz } \\
\text { fresco+alimento }\end{array}$ & $\mathrm{G}$ & 800,00 & 1,40 & Elizondo-Salazar (2015) \\
\hline
\end{tabular}


Considerando que ambos trabajos evaluaron el consumo de dietas muy similares, donde se suministraron arbustivas, gramíneas y musáceas, el consumo de king grass presentó una variabilidad de casi el doble entre ambos trabajos. Luego, Rodríguez (1989) se enfocó en evaluar el consumo de arbóreas forrajeras y utilizó diez cabras lactantes de cruce nubiana con criolla con 46 días de lactancia y $42,6 \mathrm{~kg}$ de peso vivo en promedio. Este autor obtuvo consumos de $1200 \mathrm{~g} \mathrm{MS}$.animal ${ }^{-1} \cdot \mathrm{d}^{-1} \mathrm{en}$ el caso del poró y $860 \mathrm{~g} \mathrm{MS}$.animal ${ }^{-1} \cdot \mathrm{d}^{-1}$ al utilizar madero negro.

Después de estos resultados tan favorables con arbustivas, Vallejo (1995) analizó el consumo de otras variedades de forrajes ensilados, en cabras lactantes con un peso promedio de 49,7 kg. Las cabras fueron suplementadas únicamente con ensilaje de morera (Morus sp.), amapola (Malvaviscus arboreus) y jocote (Spondias purpurea), obteniendo consumos promedios de 594, 542 y $394 \mathrm{~g} \mathrm{MS}$.animal $^{-1} . \mathrm{d}^{-1}$, para morera, amapola y jocote; respectivamente.

Tiempo después Chávez (2005) también evaluó el consumo de otros forrajes ensilados como cratylia (Cratylia argentea) y sorgo forrajero (Sorghum bicolor (L.) Moench) en doce cabras no lactantes de $41,0 \mathrm{~kg}$ de peso promedio. Los tres tratamientos empleados consistieron en ensilaje de cratylia únicamente, ensilaje de cratylia con melaza y ensilaje de cratylia con sorgo forrajero y melaza. Se presentó un consumo de 92, 204 y $515 \mathrm{~g} \mathrm{MS} . a n i m a l^{-1} . \mathrm{d}^{-1}$ para los tratamientos respectivos.

Al igual que los resultados obtenidos con king grass, el consumo de forrajes ensilados presentó una gran variabilidad y no llegaron ni al 50\% del consumo obtenido por Rodríguez (1989). Considerando que el consumo de MS reportado en los estudios fue muy bajo, la investigación en cabras continúo analizando otras fuentes forrajeras. Elizondo-Salazar (2004a,b, 2005) midió el consumo en ensayos con jaulas individuales, donde se emplearon cabras secas con peso promedio de $38,0 \mathrm{~kg}$, de la raza LaMancha, alimentadas con morera (Morus alba), ramio (Bohemeria nivea) y sorgo negro forrajero (Sorghum almum). Se evaluaron las mezclas de estos materiales, las edades de rebrote y la concentración de proteína cruda, que suplía cada uno de estos forrajes. La mayor ingesta de materia seca la presentaron los tratamientos que utilizaron morera con $737,32 \mathrm{~g} \mathrm{MS}$.animal ${ }^{-1} . \mathrm{d}^{-1}$ y la mezcla de sorgo negro forrajero y ramio con $625,88 \mathrm{~g} \mathrm{MS}$.animal ${ }^{-1} \cdot \mathrm{d}^{-1}$. Al emplear sorgo negro forrajero como ingrediente único de la dieta, no se cubrieron los requerimientos de proteína cruda para mantenimiento, por lo que se recomendó su uso como complemento en dietas ricas en proteína cruda. 
En los trabajos efectuados por Elizondo-Salazar (2004a,b, 2005), únicamente se suministró el forraje sin ningún suplemento alimenticio, presentándose una mejoría con respecto a los trabajos realizados con ensilajes. Sin embargo, seguían siendo valores bajos de consumo. Considerando que el nivel de ofrecimiento pudo haber sido un atenuante del consumo, VargasRodríguez (2009) evaluó el consumo de pasto prodigioso (Trypsacum laxum), para medir el impacto del nivel de ofrecimiento sobre el consumo en cabras. La gramínea se ofreció entera, en tres cantidades crecientes, representadas como MS con base en el peso corporal $(7,5 ; 10,0$ y $12,5 \%$ a cabras secas LaMancha, con 40,0 kg de peso, obteniendo 342,03 g MS.animal ${ }^{-1} . \mathrm{d}^{-1}$, como consumo promedio para los tres niveles de ofrecimiento. El mayor consumo de MS fue de 415,14 $\mathrm{g} \mathrm{MS}$.animal $^{-1} \cdot \mathrm{d}^{-1} \mathrm{y}$ tal como era de esperar se presentó al ofrecer la mayor cantidad de pasto $(12,5 \%)$.

En otros trabajos realizados, se consideró suplementar la dieta forrajera con alimentos balanceados, probablemente para cubrir los requerimientos que el forraje no satisface y así no tener ningún desbalance nutricional, que afectara negativamente el consumo. De esta manera, Chacón (2008), evaluó el consumo de king grass suplementado con alimento balanceado (1000 $\mathrm{g}$ en fresco. animal $\left.{ }^{-1} \cdot \mathrm{d}^{-1}\right)$. Además, evaluó el consumo de este forraje, comparando tres edades de corte (60, 75 y 90 días de rebrote) en cabras secas de razas LaMancha, Toggemburg y Saanen, con un peso promedio de $44,0 \mathrm{~kg}$. En este caso, el consumo del forraje solamente llegó a un promedio de 250,92 $\mathrm{g} \mathrm{MS}$.animal $^{-1} \cdot \mathrm{d}^{-1}$.

Herrera-Campos et al. (2009) determinó el consumo en cabras LaMancha, con un peso promedio de 50,0 kg, divididas en tres grupos y alimentadas con alimento balanceado (1000 g en fresco. animal ${ }^{-1} . \mathrm{d}^{-1}$ ) y tres diferentes forrajes (estrella africana, morera y sorgo negro forrajero). El consumo de forraje fue de $760 \mathrm{~g} \mathrm{MS}$.animal ${ }^{-1} \cdot \mathrm{d}^{-1}$ en estrella africana, $690 \mathrm{~g}$ MS.animal ${ }^{-1} \cdot \mathrm{d}^{-1}$ en sorgo negro forrajero y $630 \mathrm{~g} \mathrm{MS}$.animal $^{-1} \cdot \mathrm{d}^{-1}$ en morera.

Generalmente, los forrajes en el trópico presentan una mayor proporción de pared celular que los hace menos digestibles. Además, esto se combina con un mayor contenido de humedad en relación con forrajes de zonas templadas, lo que al final afecta negativamente el consumo, ya que un menor contenido de materia seca en la ración se asocia con una disminución en el consumo. En este sentido, Lahr et al. (1983) analizaron diferentes raciones con variaciones en el contenido de humedad y encontraron que aquellas que presentaban un porcentaje de humedad mayor al 60 ó $65 \%$ reducían el consumo considerablemente. 
Por esta razón, una manera viable para aumentar el consumo de materia seca en rumiantes podría ser el empleo de fuentes forrajeras con mayor contenido de esta. La deshidratación parcial es una forma de eliminar parte de la humedad en los forrajes sin recurrir a tratamientos físicos o químicos que aumenten la estructura de costos. En cabras, esta sencilla técnica podría aumentar el consumo y por lo tanto, mejorar la eficiencia en los sistemas de corte y acarreo que son los más difundidos actualmente en las explotaciones caprinas.

Sin embargo, Rodríguez-Zamora y Elizondo-Salazar (2012) evaluaron la calidad, selección y consumo de morera (Morus alba) y estrella africana (Cynodon nlemfuensis) de forma fresca y parcialmente deshidratados en doce cabras de las razas Saanen, Toggenburg y LaMancha, no lactantes y no gestantes, con un peso vivo promedio de $37,0 \pm 5,0 \mathrm{~kg}$. Cada animal fue ubicado en una jaula individual y recibió el forraje diariamente en una relación de $13 \%$ de su peso vivo como forraje verde. Al analizar el consumo de MS, los autores encontraron que el mayor consumo de MS se presentó en los forrajes frescos, en comparación con los deshidratados.

Elizondo-Salazar (2015) llevó a cabo un experimento con el fin de evaluar la calidad y consumo de forraje de maíz y pasto estrella africana con o sin alimento balanceado en cabras. El forraje cosechado fue suministrado a doce cabras de las razas Saanen, Toggenburg y LaMancha no lactantes y no gestantes, con un peso vivo promedio de $57,0 \pm 2,0 \mathrm{~kg}$, distribuidas en un diseño irrestricto al azar con un arreglo factorial $2 \times 2$, donde se evaluó el tipo de forraje: maíz y estrella africana y el uso o no de alimento balanceado. Los consumos fueron de 0,49;0,80;0,78 y 0,93 $\mathrm{kg} \mathrm{MS.animal}{ }^{-1} \cdot \mathrm{d}^{-1}$ para el forraje de maíz solo, forraje de maíz con alimento balanceado, pasto estrella africana solo y pasto estrella africana con alimento balanceado, respectivamente.

Más recientemente, Salazar-Acosta y Elizondo-Salazar (2017) llevaron a cabo un estudio con el objetivo de determinar el consumo de nutrientes provenientes de pasto estrella africana (Cynodon nlemfuensis) en cabras lactantes. El forraje cosechado fue suministrado sin picar a 28 cabras lactantes de las razas Saanen, Toggenburg, LaMancha y Nubiana, con un peso promedio de 52,3 $\pm 2,5 \mathrm{~kg}$. En este ensayo, los animales consumieron en promedio 0,491 kg MS.animal ${ }^{-1} \cdot \mathrm{d}^{-1}$.

Finalmente y con el objetivo de evaluar diferentes fuentes y concentraciones de saborizantes sobre el consumo de forraje en cabras, Vargas-Rodríguez y Carvajal-Villalta (2018) concluyeron que el consumo promedio de forraje en base seca que mostraron los animales cuando se aplicó un sabor ácido fue de $0,977 \mathrm{~kg} \cdot \mathrm{d}^{-1}$, mientras que, cuando se usaron saborizante dulce y salado fue de 0,855 y $0,822 \mathrm{~kg} \cdot \mathrm{d}^{-1}$, respectivamente. En dicho estudio, los investigadores utilizaron diez 
animales de las razas Saanen, Toggenburg y LaMancha, con un peso promedio de 50,0 \pm 2,1 kg y fueron alimentados con pasto king grass (Pennisetum purpureum), cosechado a 75 días de rebrote.

Considerando los estudios presentados, el consumo de materia seca en proporción al peso vivo varió desde $0,57 \%$ con king grass hasta $3,16 \%$ con morera fresca.

Todos estos valores, reportados en la literatura, no precisan claramente el panorama en cuanto al consumo de MS en cabras. Es importante tener presente que dada la deficiente calidad nutricional de muchos forrajes y el bajo consumo que se puede dar, muchas de las explotaciones caprinas en Costa Rica, no llenan los requerimientos nutricionales de las cabras y de ahí que se presentan bajas ganancias de peso y bajos niveles de producción láctea.

Es muy difícil poder establecer niveles adecuados de consumo y muchas veces no es claro definir por qué unos animales consumen más que otros. Lo que si hay que tener presente es que existe una gran variabilidad entre individuos y cuando los consumos de MS sean bajos, se debe asegurar que los animales estén consumiendo los nutrimentos necesarios para satisfacer sus necesidades de mantenimiento y producción.

Por esta razón, en toda explotación caprina se debe asegurar el ofrecimiento de un forraje de buena calidad. Sin embargo, por los bajos consumos que se presentan, en muchas ocasiones, puede ser necesario el uso de suplementos alimenticios, que ayuden a incrementar el consumo de nutrientes por estos animales.

\section{CONSIDERACIONES FINALES}

Las cabras han sido útiles para el hombre y presentan una gran adaptabilidad a condiciones ambientales variables. En muchas ocasiones presentan bajos rendimientos productivos y reproductivos a causa de una deficiente alimentación. El consumo de materia seca, especialmente de aquella proveniente del forraje, es muy variable. En Costa Rica se han encontrado rangos que van desde 250 hasta 1200 gramos por animal por día. Considerando la baja calidad nutricional de muchos de nuestros forrajes, es muy probable que con estos consumos los animales no estén llenando sus necesidades nutricionales, por lo que se deben buscar alternativas alimenticias y medidas de manejo que logren aumentar los consumos de materia seca y por ende el consumo de nutrimentos en estos animales, de manera que puedan mejorar tanto su desempeño productivo como reproductivo. 


\section{LITERATURA CITADA}

Allen, M.S. 1996. Physical constraints on voluntary intake of forages by ruminants. J. Anim. Sci. 74:3063-3075.

Baumont, R., S. Prache, M. Meuret, P. Morand. 2000. How forage characteristics influence behaviour and intake in small ruminants: a review. Lives. Prod. Sci. 64:15-28.

Burns, J., H. Mayland, D. Fisher. 2005. Dry matter intake and digestion of alfalfa harvested at sunset and sunrise. J. Anim. Sci. 83:262-270.

Castro, A. 1989. Producción de leche de cabras alimentadas con (Pennisetum purpureum x P. typhoides), suplementadas con diferentes niveles de poró (Erythrina poeppigiana) y de fruto de plátano (Musa sp. Cv. 'Pelipita'). Tesis para optar por el grado de Mag. Sc. en Ciencias Agrícolas y Recursos Naturales. CATIE. Turrialba, Costa Rica. p. 53.

Chacón, P. 2008. Estimación del aprovechamiento en ganado caprino del Pennisetum purpureum cv. king grass cosechado a tres diferentes edades de rebrote. Tesis para optar por el título de Licenciado en Ingeniería Agronómica con énfasis en Zootecnia. Universidad de Costa Rica. San José, Costa Rica. p.135.

Cháves, Q. 2005. Calidad y consumo de mezclas de Cratylia argentea y sorgo forrajero (Sorghum bicolor (L.) Moench) con y sin melaza, ensiladas en bolsas plásticas. Tesis para optar por el título de Licenciado en Ingeniería Agronómica con énfasis en Zootecnia. Universidad de Costa Rica. San José, Costa Rica. p. 56.

Devendra, C. 1981. The goat in the humid tropics. In: Goat production. (Ed. C. Gall). Academic Press, Inc. NY, USA. p. 557.

Elizondo, J. 2004a. Calidad nutricional y consumo de morera (Morus alba), ramio (Bohemeria nivea (L) GAUD) y sorgo negro forrajero (Sorghum almum) en cabras. Agronomía Mesoamericana. 15(2):209-213. 
Elizondo-Salazar, J.A. 2004b. Consumo de sorgo negro forrajero (Sorghum almum) en cabras. Agronomía Mesoamericana. 15(1):77-80.

Elizondo, J. 2005. Calidad y consumo de sorgo negro forrajero (Sorghum almum), ramio (Bohemeria nivea (L) Gaud) y mezcla de ambos. Pastos y Forrajes. 28(3):247-252.

Elizondo-Salazar, J.A. 2015. Calidad nutricional y consumo de forraje de maíz (Zea mays) y forraje de estrella africana (Cynodon nlemfuensis) con o sin alimento balanceado. Nutrición Animal Tropical. 9(2):11-26.

Fisher, D., H. Mayland, J. Burns. 1999. Variation in ruminants preference for tall fescue hays cut either at sundown or at sunup. J. Anim. Sci. 77:762-768.

Fisher, D., H. Mayland, J. Burns. 2002. Variation in ruminant preference for alfalfa hays cut at sunup and sundown. Crop. Sci. 42:231-237.

Gutiérrez, R. 1985. Utilización del follaje de poró (Eritrina poeppigiana) en combinación con banano (Musa sp. Cv. "Cavendish") como suplemento al pasto king grass (Pennisetum purpureum $x$ P. typhoides) en cabras lecheras estabuladas. Informe de trabajo especial para optar al grado de Mag. Sc, CATIE. Turrialba, Costa Rica. p.15.

Herrera-Campos, H., F. Vargas-Rodríguez, C. Boschini-Figueroa, A. Chacón-Villalobos. 2009. Variación bromatológica de la leche de cabras LaMancha alimentadas con diferentes forrajes. Agronomía Mesoamericana. 20(2):381-390.

INEC (Instituto Nacional de Estadística y Censos). 2015. VI Censo Nacional Agropecuario: Resultados Generales. Instituto de Estadística y Censos. Sector Agropecuario.

Jung, H.G., M.S Allen. 1995. Characteristics of plant cell walls affecting intake and digestibility of forages by ruminants. J. Anim. Sci. 73(9):2774-2790.

Lahr, D.A., D.E. Otterrby, D.G. Johnson, J.G. Linn, R.G. Lundquist. 1983. Effects of moisture content of complete diets on feed intake. J. Dairy Sci. 66:1891-1900. 
Morand-Fehr, P. 1981. Nutrition and feeding of goats: Application to temperate climatic conditions. In: Goat production. (Ed. Gall, C.). Academic Press, Inc. NY, USA. p.193.

Pasha, T.N., E.C. Prigge, R.W. Russel, W.B. Bryan. 1994. Influence of moisture content of forage diets on intake and digestion by sheep. J. Anim. Sci. 72:2455-2463.

Rodríguez, Z. 1989. Producción de leche de cabras alimentadas con dos especies forrajeras arbóreas: poró (Erythrina poeppigiana) y madero negro (Gliricidia sepium) suplementadas con plátano pelipita (Musa sp. Cv. 'Pelipita'). Tesis para optar por el título de Licenciada en Ingeniería Agronómica con énfasis en Zootecnia. Universidad de Costa Rica. San José, Costa Rica. p.75.

Rodríguez-Zamora, J., J.A. Elizondo-Salazar. 2012. Calidad nutricional, consumo y digestibilidad aparente de morera (Morus alba) y pasto estrella (Cynodon nlemfuensis) en cabras. Agronomía Costarricense. 33(1):13-23.

Salazar-Acosta, E., J.A. Elizondo-Salazar. 2017. Evaluación de la calidad y el consumo de pasto estrella africana por caprinos en una explotación comercial. Nutrición Animal Tropical. 11(2):61-73.

Samur, R. 1984. Producción de leche de cabras alimentadas con king grass (Pennisetum purpureum) y poró (Erythrina poeppigiana), suplementadas con fruto de banano (Musa sp. Cv. 'Cavendish'). Tesis para optar por el grado de Mag. Sc. en Ciencias Agrícolas y Recursos Naturales. CATIE. Turrialba, Costa Rica. p. 51.

Sands, M.W. 1983. Consumo de arbustos por los caprinos. In: Curso intensivo de producción caprina. CATIE, Turrialba, Costa Rica. p. 87.

Vallejo, M.A. 1995. Efecto del premarchitado y la adición de melaza sobre la calidad del ensilaje de diferentes follajes de árboles y arbustos tropicales. Tesis para optar por el título de Magister Scientiae. Centro Agronómico Tropical de Investigación y Enseñanza. Turrialba. Costa Rica. p. 115. 
Vargas-Rodríguez, C.F. 2009. Consumo y calidad del forraje Trypsacum laxum de un año de edad en cabras. Agronomía Mesoamericana. 20(2):391-398.

Vargas-Rodríguez, C.F., J.A. Carvajal-Villalta. 2018. Influencia de fuentes y concentraciones de saborizantes sobre el consumo de forraje en caprinos. Agronomía Mesoamericana. 29(2):535-545. 